\title{
The complexity of the four colour theorem
}

\author{
Cristian S. Calude and Elena Calude
}

To Professor S. Marcus on his 85th anniversary

\begin{abstract}
The four colour theorem states that the vertices of every planar graph can be coloured with at most four colours so that no two adjacent vertices receive the same colour. This theorem is famous for many reasons, including the fact that its original 1977 proof includes a non-trivial computer verification. Recently, a formal proof of the theorem was obtained with the equational logic program Coq [G. Gonthier, 'Formal proof-the four color theorem', Notices of Amer. Math. Soc. 55 (2008) no. 11, 1382-1393]. In this paper we describe an implementation of the computational method introduced by C. S. Calude and co-workers [Evaluating the complexity of mathematical problems. Part 1', Complex Systems 18 (2009) 267-285; A new measure of the difficulty of problems', J. Mult. Valued Logic Soft Comput. 12 (2006) 285-307] to evaluate the complexity of the four colour theorem. Our method uses a Diophantine equational representation of the theorem. We show that the four colour theorem is in the complexity class $\mathfrak{C}_{U, 4}$. For comparison, the Riemann hypothesis is in class $\mathfrak{C}_{U, 3}$ while Fermat's last theorem is in class $\mathfrak{C}_{U, 1}$.
\end{abstract}

\section{Introduction}

The four colour theorem, first conjectured in 1853 by Francis Guthrie, states that every plane separated into regions may be coloured using no more than four colours in such a way that no two adjacent regions receive the same colour. Two regions are called adjacent if they share a border segment, not just a point; regions must be contiguous, that is, the plan has no exclaves.

In graph-theoretical terms, the four colour theorem states that the vertices of every planar graph can be coloured with at most four colours so that no two adjacent vertices receive the same colour. In short, every planar graph is four-colourable.

The theorem was proved in $1977[\mathbf{1}, \mathbf{2}]$ (see also [17]) using a computer-assisted proof which consists of the construction of a finite set of 'configurations', and the verification that each of them is 'reducible', which implies that no configuration with this property can appear in a minimal counterexample to the theorem. Checking the correctness of the original proof is a very difficult task: it implies, among other things, checking the inputting of the descriptions of 1476 graphs, checking the correctness of the programs, proving the correctness of the compiler used to compile the programs and checking the degree of reliability of the hardware used to ran the programs. (This computer-assisted proof generated many mathematical and philosophical discussions around the notion of acceptable mathematical proof, see for example $[\mathbf{3}, \mathbf{8}, \mathbf{9}]$.) Various partial independent verifications have been obtained (it appears that there is no verification in its entirety) culminating with the formal confirmation announced in $[\mathbf{1 6}]$ which uses the equational logic program Coq (see [14] for a recent presentation of the formal proof). The following part of the concluding discussion in $[\mathbf{1 6}]$ is relevant for the current status of the proof.

Received 15 October 2009.

2000 Mathematics Subject Classification 03D15 (primary), 11 Y16 (secondary).

This work was supported in part by The Andrea von Braun Foundation, Munich, under the grant for 'Artistic Forms and Complexity'. 
However, an argument can be made that our 'proof' is not a proof in the traditional sense, because it contains steps that can never be verified by humans. In particular, we have not proved the correctness of the compiler we compiled our programs on, nor have we proved the infallibility of the hardware we ran our programs on. These have to be taken on faith, and are conceivably a source of error... .

However, from a practical point of view, the chance of a computer error that appears consistently in exactly the same way on all runs of our programs on all the compilers under all the operating systems that our programs run on is infinitesimally small compared to the chance of a human error during the same amount of case-checking.

Apart from this hypothetical possibility of a computer consistently giving an incorrect answer, the rest of our proof can be verified in the same way as traditional mathematical proofs. We concede, however, that verifying a computer program is much more difficult than checking a mathematical proof of the same length ${ }^{\dagger}$.

The four colour property is mainly of mathematical interest: K. May, quoted in [17, p. 2], says that 'Maps utilising only four colours are rare, and those that do usually require only three. Books on cartography and the history of mapmaking do not mention the four-colour property'.

We describe an implementation of the computational method, motivated by its semiotic dimension [15], introduced in $[\mathbf{5}, \mathbf{7}]$ to evaluate the complexity of the four colour theorem. Our method uses a Diophantine equational representation of the theorem. We show that the four colour theorem is in the complexity class $\mathfrak{C}_{U, 4}$; the Riemann hypothesis and the Fermat last theorem are in the lower complexity classes $\mathfrak{C}_{U, 3}$ and $\mathfrak{C}_{U, 1}$, respectively.

\section{A Diophantine equational representation of the four colour property}

We use the Diophantine representation of the four colour theorem proposed in [13], that is, a Diophantine equation

$$
F(n, t, a, \ldots)=0,
$$

such that (2.1) has no solution if and only if every planar graph can be coloured with at most four colours so that no two adjacent vertices receive the same colour.

Actually, it is better to use a pre-Diophantine representation given by the following conditions. Without restricting the generality we consider all maps $T_{n}$ consisting of the points $(x, y)$ such that $J(x, y) \leqslant Q=\left(n^{2}+3 n\right) / 2$, where $J$ is Cantor's bijection $J(x, y)=$ $\left((x+y)^{2}+3 x+y\right) / 2$. Given a four-colouring of $T_{n}, t_{0}, t_{1}, \ldots, t_{Q}$ there exist (and can be effectively computed) $s, t$ such that, for all $0 \leqslant i \leqslant Q$,

$$
t_{i}=\operatorname{rem}(t, 1+s(i+1))
$$

where the integer remainder function is denoted by rem. In other words, the sequence $t_{0}, t_{1}, \ldots, t_{Q}$ can be coded by $s$ and $t$.

Every sequence $u_{0}, u_{1}, \ldots, u_{Q}$ with $u_{i}<4$ can be represented by some $u \leqslant R=(1+4(Q+$ $2) !)^{Q+1}$ such that

$$
u_{i}=\operatorname{rem}(u, 1+4(Q+2) !(i+1)) .
$$

Finally, there is a map ( say $T_{n}$ ) which cannot be coloured with four colours if and only if the following condition is satisfied:

$$
(\exists n, t, s)(\forall u \leqslant R)(\exists x, y)(x+y \leqslant n)[A(x, y) \vee B(x, y)],
$$

\footnotetext{
†Our emphasis.
} 
where

$$
\begin{aligned}
A(x, y)= & u_{J(x, y)} \geqslant 4, \\
B(x, y)= & {\left[\left(t_{J(x, y)}=t_{J(x+1, y)} \wedge u_{J(x, y)} \neq u_{J(x+1, y)}\right)\right.} \\
& \vee\left(t_{J(x, y)} \neq t_{J(x+1, y)} \wedge u_{J(x, y)}=u_{J(x+1, y)}\right) \\
& \vee\left(t_{J(x, y)}=t_{J(x, y+1)} \wedge u_{J(x, y)} \neq u_{J(x, y+1)}\right) \\
& \left.\vee\left(t_{J(x, y)} \neq t_{J(x, y+1)} \wedge u_{J(x, y)}=u_{J(x, y+1)}\right)\right] .
\end{aligned}
$$

A simple inspection shows that the above condition is computable, so the four colour theorem is of the form $\forall n P(n)$, where $P$ is a computable predicate, that is, a $\Pi_{1}$-problem.

\section{The method}

We use a fixed 'universal formalism' for programs, more precisely, a universal self-delimiting Turing machine $U[4]$; the machine $U$ will be fully described in the next section. The machine $U$ has to be minimal in the sense that none of its instructions can be simulated by a program for $U$ written with the remaining instructions.

To every $\Pi_{1}$-problem $\pi=\forall \sigma P(\sigma)$ we associate the algorithm $\Pi_{P}=\inf \{n: P(n)=$ false $\}$ which systematically searches for a counterexample for $\pi$. There are many programs (for $U)$ which implement $\Pi_{P}$; without loss of generality, any such program will be denoted also by $\Pi_{P}$. Note that $\pi$ is true if and only if $U\left(\Pi_{P}\right)$ never halts.

The complexity (with respect to $U$ ) of a $\Pi_{1}$-problem $\pi$ is defined by the length of the smallestlength program (for $U$ ) $\Pi_{P}$, defined as above, where minimisation is calculated for all possible representations of $\pi$ as $\pi=\forall n P(n)$ :

$$
C_{U}(\pi)=\min \left\{\left|\Pi_{P}\right|: \pi=\forall n P(n)\right\} .
$$

For $C_{U}$ it is irrelevant whether $\pi$ is known to be true or false. In particular, the program containing the single instruction halt is not a $\Pi_{P}$ program, for any $P$.

Because the complexity $C_{U}$ is incomputable, we can work only with upper bounds for $C_{U}$. As the exact value of $C_{U}$ is not important, following [6] we classify $\Pi_{1}$-problems into the following classes:

$$
\mathfrak{C}_{U, n}=\left\{\pi: \pi \text { is a } \Pi_{1} \text {-problem, } C_{U}(\pi) \leqslant n \text { kbit }\right\}
$$

(a kilobit (kbit or $\mathrm{kb}$ ) is equal to $2^{10}$ bits).

As the four colour theorem is a $\Pi_{1}$-problem, we choose a specific representation $4 \mathrm{CT}=$ $\forall \sigma P(\sigma)$, and based on it we write the program $\Pi_{4 \mathrm{CT}}$, optimise it in length, and finally use the size of the program $\left|\Pi_{4 \mathrm{CT}}\right|$ as an upper bound on $C_{U}(4 \mathrm{CT})$. We found that the four colour theorem is in the class $\mathfrak{C}_{U, 4}$.

\section{A universal prefix-free binary Turing machine}

We briefly describe the syntax and the semantics of a register machine language which implements a (natural) minimal universal prefix-free binary Turing machine $U$; it is a refinement, constructed in $[6]$, of the languages in $[\mathbf{7}, \mathbf{1 0}, \mathbf{1 2}]$.

Any register program (machine) uses a finite number of registers, each of which may contain an arbitrarily large non-negative integer.

By default, all registers, named with a string of lower or upper case letters, are initialised to 0 . Instructions are labeled by default with $0,1,2, \ldots$.

The register machine instructions are listed below. Note that in all cases R2 and R3 denote either a register or a non-negative integer, while $\mathrm{R} 1$ must be a register. When referring to $\mathrm{R}$ we use, depending upon the context, either the name of register $\mathrm{R}$ or the non-negative integer stored in R. 


\section{$=\mathbf{R} 1, \mathbf{R} 2, \mathbf{R} 3$}

If the contents of R1 and R2 are equal, then the execution continues at the R3th instruction of the program. If the contents of $\mathrm{R} 1$ and $\mathrm{R} 2$ are not equal, then execution continues with the next instruction in sequence. If the content of R3 is outside the scope of the program, then we have an illegal branch error.

\section{$\& \mathbf{R} 1, \mathbf{R 2}$}

The contents of register $\mathrm{R} 1$ is replaced by $\mathrm{R} 2$.

\section{$+\mathbf{R} 1, \mathbf{R 2}$}

The contents of register R1 is replaced by the sum of the contents of $\mathrm{R} 1$ and $\mathrm{R} 2$.

\section{!R1}

One bit is read into the register R1, so the contents of $\mathrm{R} 1$ becomes either 0 or 1 . Any attempt to read past the last data-bit results in a run-time error.

\section{$\%$}

This is the last instruction for each register machine program before the input data. It halts the execution in two possible states: either it successfully halts or it halts with an under-read error.

A register machine program consists of a finite list of labeled instructions from the above list, with the restriction that the halt instruction appears only once, as the last instruction of the list. The input data (a binary string) follows immediately after the halt instruction. A program not reading the whole data or attempting to read past the last data-bit results in a run-time error. Some programs (such as the ones presented in this paper) have no input data; these programs cannot halt with an under-read error.

The instruction $=\mathrm{R}, \mathrm{R}, \mathrm{n}$ is used for the unconditional jump to the $n$th instruction of the program. For Boolean data types we use integers $0=$ false and $1=$ true.

For longer programs it is convenient to distinguish between the main program and some sets of instructions called 'routines' which perform specific tasks for another routine or the main program. The call and call-back of a routine are executed with unconditional jumps.

\section{Binary coding of programs}

To compute an upper bound on $C_{U}(4 \mathrm{CT})$ we need to compute the size in bits of the program $\Pi_{4 \mathrm{CT}}$, so we need to uniquely code in binary the programs for $U$. To this aim we use a prefix-free coding as follows.

The binary coding of special characters (instructions and comma) is shown in Table 1 ( $\varepsilon$ is the empty string).

For registers we use the prefix-free code code $1=\left\{0^{|x|} 1 x \mid x \in\{0,1\}^{*}\right\}$. The codes of the first 32 registers are shown in Table 2 . The register names are chosen to optimise the length of the program, that is, the most frequent registers have the smallest code ${ }_{1}$ length.

For non-negative integers we use the prefix-free code code $e_{2}=\left\{1^{|x|} 0 x \mid x \in\{0,1\}^{*}\right\}$. The codes of the first 16 non-negative integers are shown in Table 3 (as $x \varepsilon=\varepsilon x=x$, for every string $x \in\{0,1\}^{*}$, in what follows we omit $\varepsilon$ ).

TABLE 1. The binary coding of special characters.

\begin{tabular}{cccc}
\hline Special characters & Code & Special characters & Code \\
\hline$\&$ & $\varepsilon$ & + & 111 \\
$\&$ & 01 & $!$ & 110 \\
$=$ & 00 & $\%$ & 100 \\
\hline
\end{tabular}


The instructions are coded by self-delimiting binary strings as follows.

(1) \&R1, R2 is coded in two different ways depending on R2:

$$
01 \operatorname{code}_{1}(\mathrm{R} 1) \operatorname{code}_{i}(\mathrm{R} 2) \text {, }
$$

where $i=1$ if $\mathrm{R} 2$ is a register and $i=2$ if $\mathrm{R} 2$ is an integer.

(2) $+R 1, R 2$ is coded in two different ways depending on R2:

$$
111 \operatorname{code}_{1}(\mathrm{R} 1) \operatorname{code}_{i}(\mathrm{R} 2),
$$

where $i=1$ if $\mathrm{R} 2$ is a register and $i=2$ if $\mathrm{R} 2$ is an integer.

(3) =R1, R2, R3 is coded in four different ways depending on the data types of R2 and R3:

$$
00 \operatorname{code}_{1}(\mathrm{R} 1) \operatorname{code}_{i}(\mathrm{R} 2) \operatorname{code}_{j}(\mathrm{R} 3),
$$

where $i=1$ if $\mathrm{R} 2$ is a register and $i=2$ if $\mathrm{R} 2$ is an integer, $j=1$ if $\mathrm{R} 3$ is a register and $j=2$ if R3 is an integer.

(4) !R1 is coded by

$$
110 \operatorname{code}_{1}(\mathrm{R} 1) \text {. }
$$

(5) $\%$ is coded by

100.

TABLE 2. The codes of the first 32 registers.

\begin{tabular}{cccccccc}
\hline Register & Code $_{1}$ & Register & Code $_{1}$ & Register & Code $_{1}$ & Register & Code $_{1}$ \\
\hline $\mathrm{R}_{1}$ & 010 & $\mathrm{R}_{9}$ & 0001010 & $\mathrm{R}_{17}$ & 000010010 & $\mathrm{R}_{25}$ & 000011010 \\
$\mathrm{R}_{2}$ & 011 & $\mathrm{R}_{10}$ & 0001011 & $\mathrm{R}_{18}$ & 000010011 & $\mathrm{R}_{26}$ & 000011011 \\
$\mathrm{R}_{3}$ & 00100 & $\mathrm{R}_{11}$ & 0001100 & $\mathrm{R}_{19}$ & 000010100 & $\mathrm{R}_{27}$ & 000011100 \\
$\mathrm{R}_{4}$ & 00101 & $\mathrm{R}_{12}$ & 0001101 & $\mathrm{R}_{20}$ & 000010101 & $\mathrm{R}_{28}$ & 000011101 \\
$\mathrm{R}_{5}$ & 00110 & $\mathrm{R}_{13}$ & 0001110 & $\mathrm{R}_{21}$ & 000010110 & $\mathrm{R}_{29}$ & 000011110 \\
$\mathrm{R}_{6}$ & 00111 & $\mathrm{R}_{14}$ & 0001111 & $\mathrm{R}_{22}$ & 000010111 & $\mathrm{R}_{30}$ & 000011111 \\
$\mathrm{R}_{7}$ & 0001000 & $\mathrm{R}_{15}$ & 000010000 & $\mathrm{R}_{23}$ & 000011000 & $\mathrm{R}_{31}$ & 00000100000 \\
$\mathrm{R}_{8}$ & 0001001 & $\mathrm{R}_{16}$ & 000010001 & $\mathrm{R}_{24}$ & 000011001 & $\mathrm{R}_{32}$ & 00000100001 \\
\hline
\end{tabular}

TABLE 3 . The codes of the first 16 non-negative integers.

\begin{tabular}{cccccccc}
\hline Integer & Code $_{2}$ & Integer & Code $_{2}$ & Integer & Code $_{2}$ & Integer & Code $_{2}$ \\
\hline 0 & 100 & 4 & 11010 & 8 & 1110010 & 12 & 1110110 \\
1 & 101 & 5 & 11011 & 9 & 1110011 & 13 & 1110111 \\
2 & 11000 & 6 & 1110000 & 10 & 1110100 & 14 & 111100000 \\
3 & 11001 & 7 & 1110001 & 11 & 1110101 & 15 & 111100001 \\
\hline
\end{tabular}

TABLE 4. The register machine routine to compute in $d$ the integer remainder of $a$ divided by $b$, for integers $a \geqslant b \geqslant 0$.

\begin{tabular}{cccc}
\hline Instruction number & Instruction & Code & Length \\
\hline 0 & \&h, e & 01000100100110 & 14 \\
1 & $\& \mathrm{e}, \mathrm{b}$ & 0100110011 & 10 \\
2 & $\& \mathrm{~d}, \mathrm{O}$ & 0100101100 & 10 \\
3 & $=\mathrm{e}, \mathrm{a}, \mathrm{B}$ & 00001100101110010 & 17 \\
4 & $+\mathrm{e}, 1$ & 11100110101 & 11 \\
5 & $+\mathrm{d}, \mathrm{1}$ & 11100101101 & 11 \\
6 & $=\mathrm{d}, \mathrm{b}, 2$ & 000010101111000 & 15 \\
7 & $=\mathrm{a}, \mathrm{a}, \mathrm{3}$ & 0001001011001 & 13 \\
8 & $\& \mathrm{e}, \mathrm{h}$ & 01001100001001 & 14 \\
9 & $=\mathrm{a}, \mathrm{a}, \mathrm{c}$ & 0001001000100 & 13 \\
\hline
\end{tabular}


All codings for instruction names and special symbol comma, registers and non-negative integers are self-delimiting; the prefix-free codes used for registers and non-negative integers are disjoint. The code of any instruction is the concatenation of the codes of the instruction name and the codes (in order) of its components; hence the set of codes of instructions is prefix-free. The code of a program is the concatenation of the codes of its instructions, so the set of codes of all programs is prefix-free too.

The smallest program which halts is 100 and the smallest program which never halts is 00010010100100 .

For example, the register machine routine in Table 4 computes in $d$ the integer remainder of $a$ divided by $b$, for integers $a \geqslant b \geqslant 0$ (if $b=0$ then $d=0$ ). Here we use $\mathrm{R}_{1}=\mathrm{a}, \mathrm{R}_{2}=\mathrm{b}$, $\mathrm{R}_{3}=\mathrm{c}, \mathrm{R}_{4}=\mathrm{d}, \mathrm{R}_{5}=\mathrm{e}, \mathrm{R}_{8}=\mathrm{h}$.

The routine can be uniquely encoded by concatenating the binary strings coding the instructions of the routine:

$$
\begin{aligned}
& 0100010010011001001100110100101100000011001011100101110011010111 \\
& 1001011010000101011110000001001011001010011000010010001001000100
\end{aligned}
$$

which is a string of length 128 bits.

\section{The program $\Pi_{4 \mathrm{CT}}$}

In this section we present the program $\Pi_{4 \mathrm{CT}}$ which is based on the computable predicate $P$ described in Section 2. In order to help the understanding, the following formulas are used in the program:

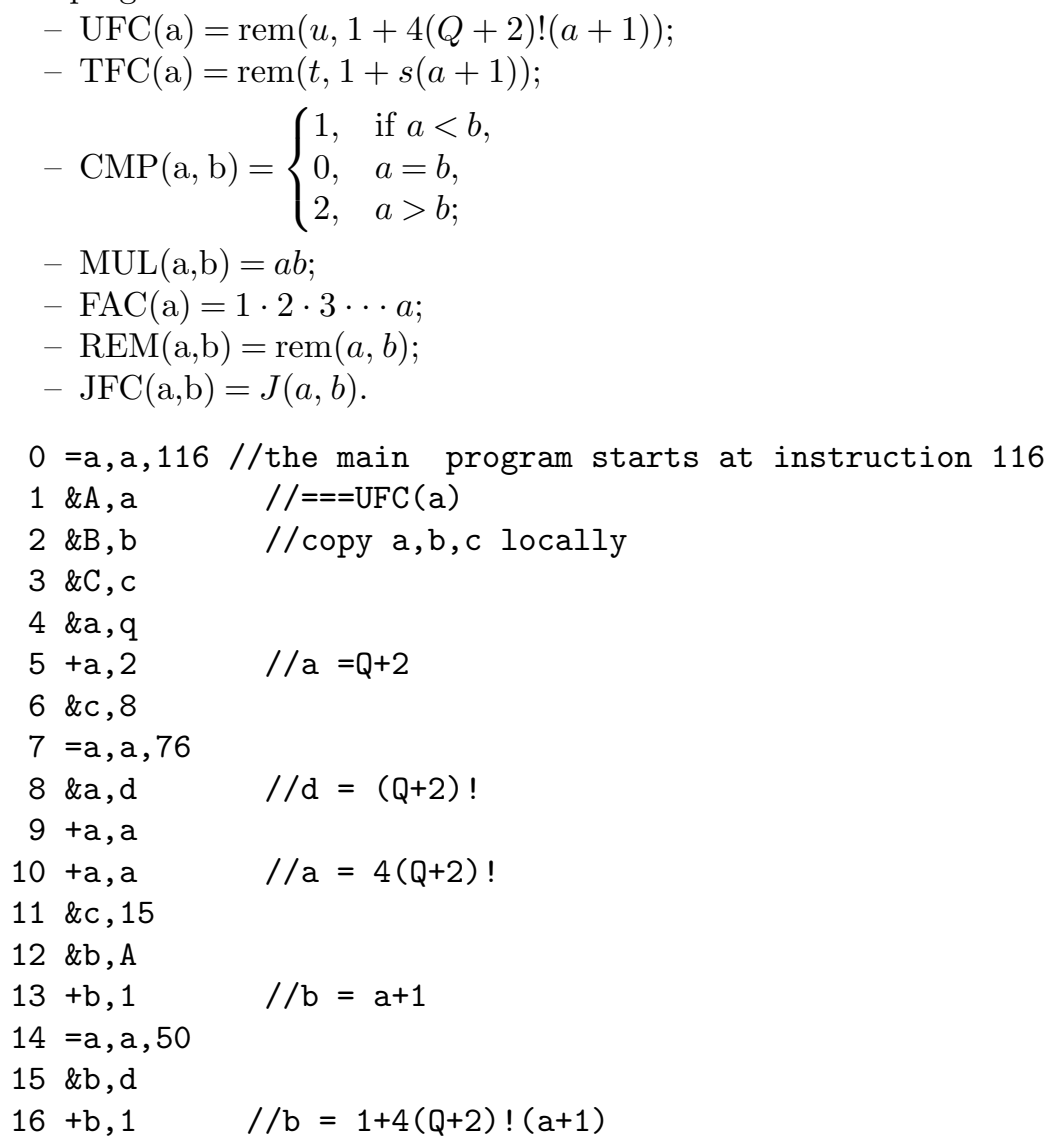


$17 \& a, u$

18 \&c, 20

$19=\mathrm{a}, \mathrm{a}, 60$

$20=\mathrm{d}, 1,27$

21 \&c, 23

$/ / \mathrm{u}<1+4(\mathrm{Q}+2) !(\mathrm{a}+1)$

$22=\mathrm{a}, \mathrm{a}, 91$

$/ / \mathrm{u}>=1+4(\mathrm{Q}+2) !(\mathrm{a}+1)$

23 \&a, A

$24 \& b, B$

$25 \& c, C$

$26=\mathrm{a}, \mathrm{a}, \mathrm{c}$

$27 \& d, a$

$28=a, a, 23$

29 \&aa, a

$30 \& b b, b$

$31 \& c c, c$

$32+a, 1$

$/ / \mathrm{d}=\operatorname{REM}(\mathrm{u}, 1+4(\mathrm{Q}+2) !(\mathrm{a}+1))$

33 \&b, s

$=a+1$

$34 \& c, 36$

$35=a, a, 50$

$/ /===\operatorname{TFC}(\mathrm{a})$

$36 \& b, d$

$37+b, 1$

$38 \& a, t$

$39 \& c, 41$

$40=a, a, 60$

$41=\mathrm{d}, 1,48$

42 \&c, 44

$/ / \mathrm{b}=\mathrm{s}$

$43=\mathrm{a}, \mathrm{a}, 91$

$/ / \mathrm{d}=(\mathrm{a}+1) \mathrm{s}$

44 \&a, aa

$45 \& b, b b$

$46 \& c, c c$

$47=a, a, c$

48 \&d, a

$/ / \mathrm{d}=\mathrm{t}$

$49=a, a, 44$

50 \&ee, $e$

$51 \& d, 0$

$52=\mathrm{b}, 0,58$

$/ / b=1+(a+1) s$

$/ / \mathrm{a}=\mathrm{t}$

53 \&e, 1

$54+d, a$

$55=e, b, 58$

$/ / d=\operatorname{CMP}(t, 1+(a+1) s)$

$/ / \mathrm{t}<1+(\mathrm{a}+1) \mathrm{s}$

$/ / \mathrm{t}>=1+\mathrm{s}(\mathrm{a}+1)$

$/ / \mathrm{d}=\operatorname{REM}(\mathrm{t}, 1+\mathrm{s}(\mathrm{a}+1))$

$56+e, 1$

$57=a, a, 54$

58 \&e, ee

$59=\mathrm{a}, \mathrm{a}, \mathrm{c}$

60 \&ee, $e$

$61 \& f f, f$

62 \&e, a

$63 \& f, b$

$64+\mathrm{e}, 1$

$65+f, 1$

66 \&d, 0

$67=e, f, 73 \quad / / a=b$ 


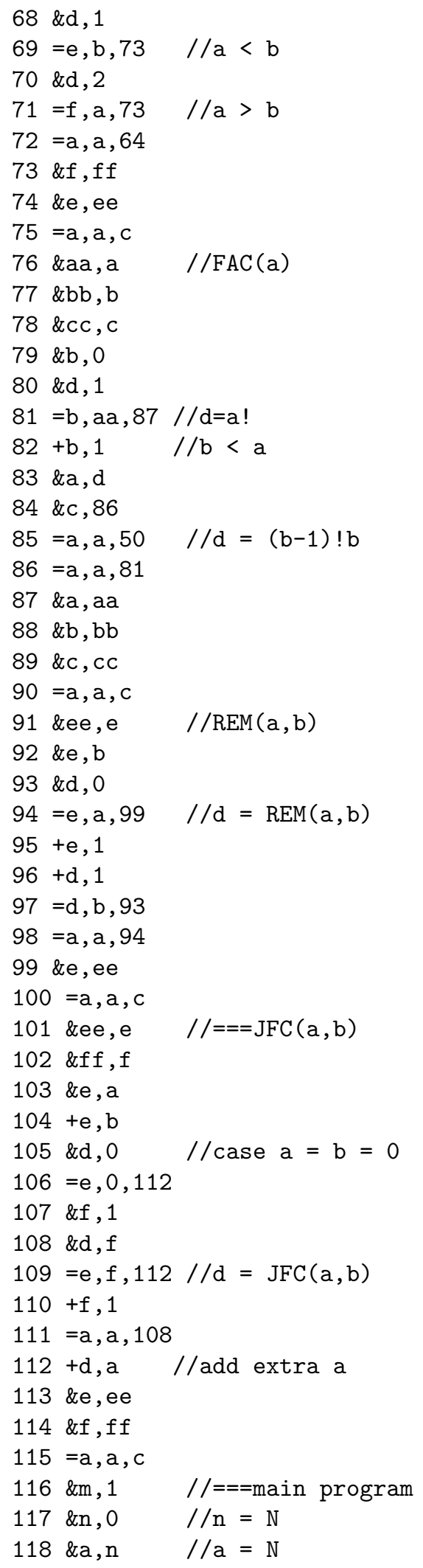




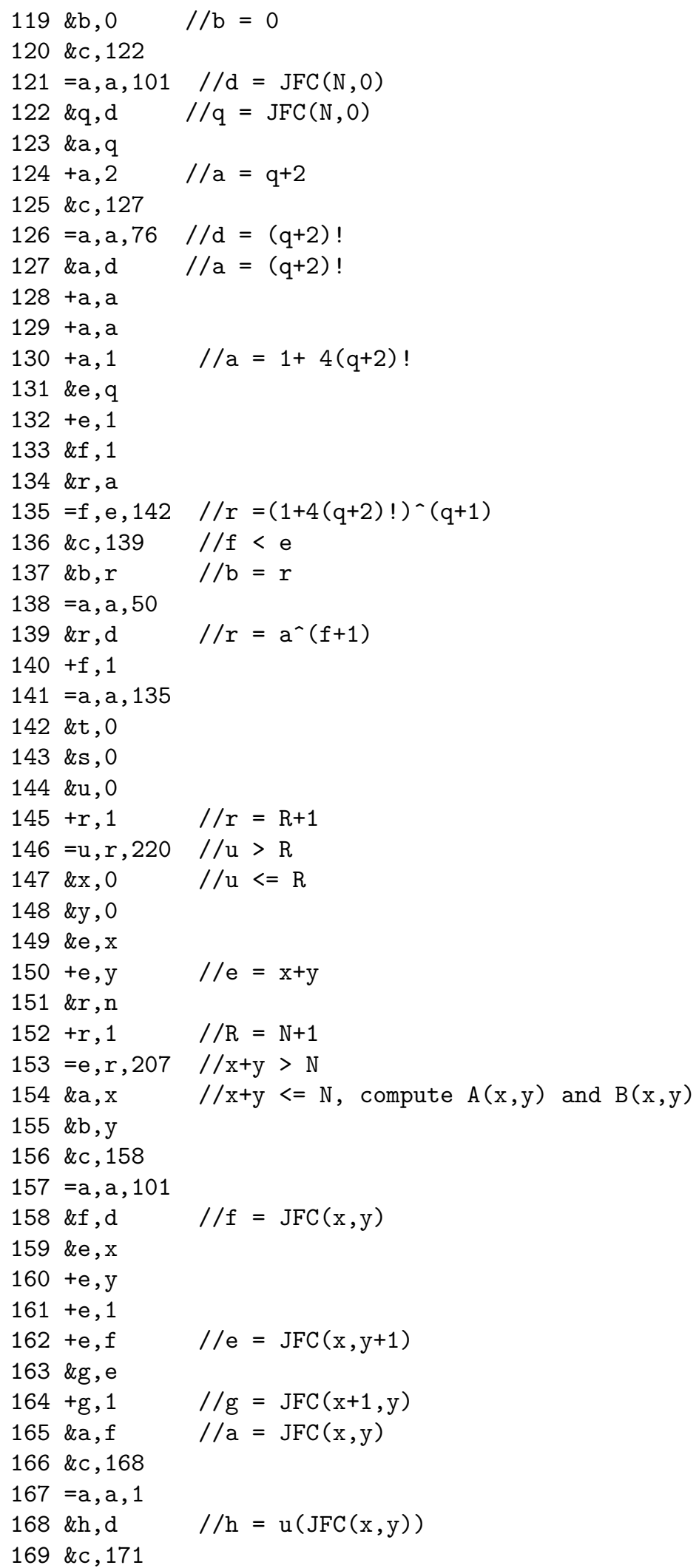




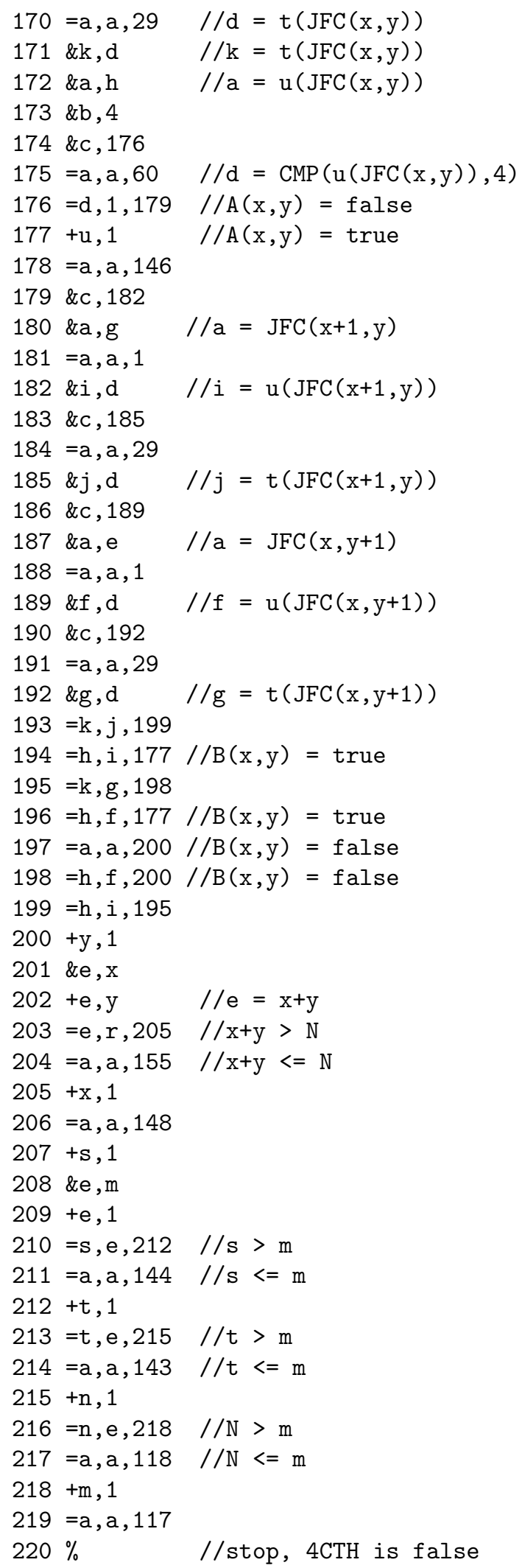


We use the following conversion table for registers.

\begin{tabular}{llllccc}
\hline $\mathrm{R}_{1}=\mathrm{a}$ & $\mathrm{R}_{2}=\mathrm{e}$ & $\mathrm{R}_{3}=\mathrm{c}$ & $\mathrm{R}_{4}=\mathrm{d}$ & $\mathrm{R}_{5}=\mathrm{b}$ & $\mathrm{R}_{6}=\mathrm{f}$ & $\mathrm{R}_{7}=\mathrm{r}$ \\
$\mathrm{R}_{8}=\mathrm{e}$ & $\mathrm{R}_{9}=\mathrm{h}$ & $\mathrm{R}_{10}=\mathrm{x}$ & $\mathrm{R}_{11}=\mathrm{y}$ & $\mathrm{R}_{12}=\mathrm{g}$ & $\mathrm{R}_{13}=\mathrm{n}$ & $\mathrm{R}_{14}=\mathrm{aa}$ \\
$\mathrm{R}_{15}=\mathrm{q}$ & $\mathrm{R}_{16}=\mathrm{s}$ & $\mathrm{R}_{17}=\mathrm{t}$ & $\mathrm{R}_{18}=\mathrm{u}$ & $\mathrm{R}_{19}=\mathrm{bb}$ & $\mathrm{R}_{20}=\mathrm{cc}$ & $\mathrm{R}_{21}=\mathrm{ff}$ \\
$\mathrm{R}_{22}=\mathrm{i}$ & $\mathrm{R}_{23}=\mathrm{k}$ & $\mathrm{R}_{24}=\mathrm{m}$ & $\mathrm{R}_{25}=\mathrm{A}$ & $\mathrm{R}_{26}=\mathrm{j}$ & $\mathrm{R}_{27}=\mathrm{B}$ & $\mathrm{R}_{28}=\mathrm{C}$ \\
\hline
\end{tabular}

Consequently, the upper bound given by the size of the program $\Pi_{4 \mathrm{CT}}$ for $U$ is 3489 bits, so the four colour theorem belongs to the class $\mathfrak{C}_{U, 4}$.

\section{Final comments}

We have shown that the four colour theorem is in $\mathfrak{C}_{U, 4}$. It is possible to decrease the size of the program $\Pi_{4 \mathrm{CT}}$ by optimising the code or by using a different computable predicate. We conjecture that the four colour theorem is not in $\mathfrak{C}_{U, 2}$.

The following mathematical statements are in lower complexity classes: Legendre's conjecture, Fermat's last theorem and Goldbach's conjecture are in $\mathfrak{C}_{U, 1}$ and the Riemann hypothesis is in $\mathfrak{C}_{U, 3}[\mathbf{6}, \mathbf{1 1}]$.

Acknowledgements. We thank Michael Dinneen and Nadia Kasto for critical comments and extensive discussions which improved this paper.

\section{References}

1. K. Appel and W. Haken, 'Every planar map is four-colorable, II: reducibility', Illinois J. Math. 21 (1977) 491-567.

2. K. Appel, W. HAKen and J. Koch, 'Every planar map is four colourable, I: discharging', Illinois J. Math. 21 (1977) 429-490.

3. A. S. Calude, 'The journey of the four colour theorem through time', NZ Math. Magazine 38 (2001) no. 3, 27-35.

4. C. S. CAlude, Information and randomness: an algorithmic perspective, 2nd edn (Springer, Berlin, 2002) revised and extended.

5. C. S. Calude and E. Calude, 'Evaluating the complexity of mathematical problems. Part 1', Complex Systems 18 (2009) 267-285.

6. C. S. Calude and E. Calude, 'Evaluating the complexity of mathematical problems. Part 2', Complex Systems 18 (2010) 387-401.

7. C. S. Calude, E. Calude and M. J. Dinneen, 'A new measure of the difficulty of problems', J. Mult. Valued Logic Soft Comput. 12 (2006) 285-307.

8. C. S. Calude, E. Calude and S. Marcus, 'Passages of proof', Bull. EATCS 84 (2004) 167-188.

9. C. S. Calude, E. Calude and S. Marcus, 'Proving and programming', Randomness \& complexity, from Leibniz to Chaitin (ed. C. S. Calude; World Scientific, Singapore, 2007) 310-321.

10. C. S. Calude, M. J. Dinneen and C.-K. Shu, 'Computing a glimpse of randomness', Experiment. Math. 11 (2002) no. 2, 369-378.

11. E. CALUDE, 'The complexity of the Goldbach's conjecture and Riemann's hypothesis', CDMTCS Research Report 369, 2009, 14 pp.

12. G. J. Chaitin, Algorithmic information theory (Cambridge University Press, Cambridge, 1987) third printing 1990.

13. M. Davis, Y. V. Matijasevič and J. Robinson, 'Hilbert's tenth problem. Diophantine equations: positive aspects of a negative solution', Mathematical developments arising from Hilbert problems (ed. F. E. Browder; American Mathematical Society, Providence, RI, 1976) 323-378.

14. G. Gonthier, 'Formal proof-the four color theorem', Notices Amer. Math. Soc. 55 (2008) no. 11, $1382-1393$

15. S. Marcus, 'Mathematics through the glasses of Hjelmslev's semiotics', Semiotica 145-1/4 (2003) $235-246$.

16. N. Robertson, D. P. Sanders, P. Seymour and R. Thomas, 'The four color theorem', http://www.math.gatech.edu/ thomas/FC/fourcolor.html (accessed 30 November 2008).

17. R. Wilson, Four colours suffice (Penguin, London, 2002). 
Cristian S. Calude

Department of Computer Science The University of Auckland

Private Bag 92019, Auckland

New Zealand

c.calude@auckland.ac.nz

http://www.cs.auckland.ac.nz/

$\sim$ cristian

\section{Elena Calude}

Institute of Information and

Mathematical Sciences

Massey University at Albany

Private Bag 102-904, North Shore MSC

New Zealand

http://www.massey.ac.nz/ ecalude 